\title{
Le barriere allo sviluppo della dialisi peritoneale non esistono
}

\author{
Roberto Dell'Aquila, Graziella Berlingò, Valentina Pellanda, Andrea Contestabile, \\ Paolo Lentini, Anna Basso
}

Struttura Complessa di Nefrologia e Dialisi, Ospedale San Bassiano, Bassano del Grappa (VI)

\begin{abstract}
THERE ARE NO BARRIERS TO THE DEVELOPMENT OF PERITONEAL DIALYSIS
Abstract. Peritoneal dialysis is a method used in Italy from the 70s; starting from the first unsuccesses due to inexperience, today we reached a point in which the technique is safe and supported by a large amount of literature. Nevertheless, the percentage of patients using this method has settled for a long time at $\mathbf{1 0 \%}$, with a negative trend in the recent years. It is not understood why such a simple and yet so effective technique fails to obtain a more respectable space. Much has been written about the so-called "barriers" to its development, among which social, economical, and organizational issues are included. The purpose of this work is to show, using examples, that these barriers are only determined by the inexperience and total lack of will of those who run a nephrology unit.
\end{abstract}

Key words: Peritoneal dialysis, Peritonitis, Exit-site, Barriers

Conflict of interest: None.

Financial support: None.

Accettato: 16 Agosto 2013

\section{Un po' di storia}

La dialisi peritoneale ha una storia lunga quasi come quella della dialisi extracorporea. La scoperta della capacità del peritoneo di agire come una membrana semipermeabile si può attribuire agli studi del tedesco Wegner che, nel 1877, pubblicò $\mathrm{i}$ risultati di una serie di esperimenti, condotti infondendo soluzioni saline nella cavità peritoneale di conigli, durante $i$ quali egli osservò un incremento nel volume delle preparazioni a concentrazione zuccherina concentrata durante il tempo di permanenza nella cavità addominale. Nel 1946, a Boston, tre medici della Mayo Clinic, Frank, Seligman e Fine, studiarono sistematicamente la composizione della soluzione dializzante e la velocità di infusione necessaria per assicurare, con la tecnica a flusso continuo, un'efficiente azione di rimozione dei soluti tossici. Negli anni successivi, furono fatti notevoli progressi nella composizione delle soluzioni di dialisi e nella tecnica di accesso al peritoneo, che portarono al successivo fondamentale passo in avanti, quello della standardizzazione della tecnica. Nel 1959, Doolan e Maxwell cominciarono a usare soluzioni di dialisi preparate industrialmente contenute in bottiglie di vetro, che venivano fatte defluire per gravità nel peritoneo attraverso una connessione con il catetere, e che, dopo un tempo di permanenza, venivano drenate dall'addome ponendo le bottiglie sul pavimento. Il sistema era semplice e facile da eseguire ma vi era un'elevata incidenza di conta- minazioni batteriche e, conseguentemente, di peritoniti. Agli inizi degli anni sessanta, la dialisi peritoneale era ormai divenuta un valido trattamento sostitutivo della funzione renale in caso di insufficienza renale acuta, da preferire soprattutto in caso di pazienti con rischi emorragici, mentre era considerata una seconda scelta come trattamento cronico. Un significativo passo avanti si ebbe con l'introduzione, nel 1968, del catetere di Tenckhoff, che costituì, e costituisce tuttora, soprattutto con le sue versioni più recenti, una pietra miliare per la sua capacità di garantire una via d'accesso permanente e affidabile al peritoneo. Nel 1978, venne pubblicato il lavoro di Popovich e Moncrief, che lanciava la tecnica manuale nella dialisi peritoneale chiamata CAPD, Continuous Ambulatory Peritoneal Dialysis.

È d'obbligo ricordare anche i ricercatori italiani che hanno dato un grosso contributo allo sviluppo della dialisi peritoneale, come, per esempio, Buoncristiani che, nel 1980, propose il "sistema Perugia", composto da un connettore a Y fissato al catetere peritoneale e chiuso da due tappi, e Bazzato, che modificò il "sistema Perugia" progettando un sistema a doppia sacca nel quale le branche a Y facevano parte integrante del set disposable; queste due soluzioni ridussero di gran lunga l'incidenza delle peritoniti. Bisogna ricordare, inoltre, De Vecchi (1) che, insieme a Scalamogna, inventò il "cuff-shaving" per salvare il catetere peritoneale dalle infezioni dell'exit-site resistenti alla terapia antibiotica, Chiaramonte, che inventò il 
catetere corto riducendo le dislocazioni, come pure fece Di Paolo con il catetere autolocante, Feriani e la sacca a doppio comparto, che permise l'utilizzo del bicarbonato come tampone, e molti altri ancora, con i quali mi scuso se non ho riportato i loro nomi e i loro lavori ma anche la mia memoria falla.

\section{Un'altra storia}

Quando lavoravo in dialisi peritoneale a Vicenza, grazie alla lungimiranza del Prof. La Greca, riuscii a portare la popolazione peritoneale da 76 a 107 , ovvero circa il $50 \%$ dei pazienti in trattamento dialitico. Nel Dicembre del 2008 arrivai a Bassano del Grappa, dove trovai 85 pazienti in emodialisi e 3 pazienti in peritoneale. Nel Febbraio del 2009, cominciai un'azione di informazione sul territorio e anche all'interno dell'Azienda Sanitaria, con il risultato, non facile, che oggi abbiamo 88 pazienti in emodialisi e 32 pazienti in dialisi peritoneale e 4 strutture residenziali sia in pianura che in montagna, che ci garantiscono 8 posti letto per pazienti non autosufficienti che vogliono continuare con la metodica peritoneale: oggi sono $\mathrm{i}$ pazienti stessi che chiedono di fare la dialisi peritoneale!

\section{Una storia americana}

Due Autori americani, Daron Acemoglu e James A. Robinson (2), che nulla hanno a che fare con la medicina e tanto meno con la dialisi peritoneale, raccontano una storia assai interessante: la cittadina di Nogales, nel 1853, fu divisa in due a causa del confine fra Stati Uniti d'America e Messico. Oggi esiste Nogales Sonora in territorio messicano e Nogales Arizona in territorio americano. A Nogales Arizona il reddito medio annuale di una famiglia è di circa 30.000 dollari, i ragazzi vanno a scuola, la maggioranza degli adulti ha un diploma di istruzione superiore e la popolazione gode di buona salute con un'alta aspettativa di vita, secondo gli standard globali; molti dei residenti hanno più di 65 anni e il diritto all'assistenza sanitaria pubblica di Medicare, hanno servizi essenziali come l'elettricità, i telefoni, un sistema fognario e una rete stradale che li collega al resto degli Stati Uniti e, infine, ma non meno importante, hanno la garanzia della legalità e dell'ordine. A Nogales Sonora, il reddito è circa un terzo rispetto a quello dei loro vicini, gran parte degli adulti non ha un diploma e molti adolescenti non vanno a scuola, vi è un alto tasso di mortalità infantile, vi è una scarsa qualità del servizio sanitario, non c'è accesso ad altri servizi pubblici, le strade sono in cattive condizioni e la legalità e l'ordine sono in una situazione ancora peggiore. Come possono le due metà di quella che è, in sostanza, la stessa città essere così diverse? E sì che non c'è differenza geografica, il clima è lo stesso e le malattie sono le stesse tipiche dell'area! La spiegazione viene data dalla differente gestione politica e amministrativa delle due metà della stessa cittadina.

\section{Conclusioni}

Cosa ci insegnano queste storie?

Da anni si scrivono articoli sulla bontà della metodica dialitica peritoneale, si fanno convegni dove si magnificano le virtù di questa metodica e si fanno questionari per cercare di capire quali sono le barriere che impediscono lo sviluppo della peritoneale; ebbene le storie appena raccontate ci fanno capire che non bisogna andare tanto lontano per capire perché due fenomeni uguali sono così diversi: se le due Nogales sono così diverse è perché chi comanda è diverso, ha idee diverse e forse interessi diversi e non capisce, o non vuole capire, che la buona salute della sua popolazione si rifletterà su un futuro risparmio. Se Vicenza ha il 50\% di pazienti in dialisi peritoneale e se a Bassano del Grappa in 4 anni siamo passati dal 3.4\% al $26.6 \%$ di prevalenza in peritoneale non è perché in queste due aree c'è un clima migliore o chissà quale strana alchimia, non è perché abbiamo abbattuto delle barriere impossibili, ma semplicemente perché abbiamo creduto in questa metodica e ci siamo impegnati a farla crescere. Si dice che la peritoneale è una Cenerentola: è vero, ma Cenerentola, in teoria, non poteva andare al ballo perché qualcuno non voleva che ci andasse!

Se tutti i Direttori di Struttura "volessero" fare la peritoneale, oggi non saremmo qui a parlare di barriere e la metodica non sarebbe di certo una tecnica "marginale", ma avrebbe la sua dignitosa collocazione che, peraltro, si merita. Penso sia ora di smetterla di nasconderci dietro un dito e di continuare a cercare cause inesistenti.

Quello che mi auguro è che, quando la carrozza di Cenerentola si trasformerà in "zucca", ci sia abbastanza sale dentro!

\section{Riassunto}

La dialisi peritoneale è una metodica in uso in Italia dagli anni '70; dalle prime sconfitte dovute all'inesperienza, siamo giunti oggi, se non ieri, a una metodica sicura e con una mole di letteratura favorevole. Malgrado ciò, la percentuale di utilizzo di tale metodica è assestata da molto tempo sul $10 \%$ e con un trend in negativo in questi ultimi anni. Non si comprende come una tecnica così semplice e, al tempo stesso, così efficace non riesca ad avere un suo rispettabile spazio. Molto è stato scritto sulle cosiddette "barriere" al suo sviluppo, tra le quali si annoverano cause sociali, economiche e organizzative. Lo scopo di questo lavoro è di dimostrare con degli esempi che, se barriere ci sono, queste sono rappresentate solo dall'inesperienza e dalla mancanza totale di volontà di chi dirige una struttura di nefrologia.

Parole chiave: Dialisi peritoneale, Peritoniti, Exit-site, Sviluppo

Dichiarazione di conflitto di interessi: Gli Autori dichiarano di non avere conflitto di interessi.

Contributi economici degli autori: Gli Autori dichiarano di non aver ricevuto sponsorizzazioni economiche per la preparazione dell'articolo. 
Indirizzo degli Autori:

Dr. Roberto Dell'Aquila

S.C. di Nefrologia e Dialisi

Ospedale San Bassiano

Via dei Lotti 40

36061 Bassano del Grappa (VI)

roberto.dellaquila@aslbassano.it

\section{Bibliografia}

1. Scalamogna A, De Vecchi A, Maccario M, Castelnovo C, Ponticelli C. Cuff-shaving procedure. A rescue treatment for exit-site infection unresponsive to medical therapy. Nephrol Dial Transplant 1995; 10 (12): 2325-7.

2. Acemoglu D, Robinson JA. Perché le nazioni falliscono. Alle origini di prosperità, potenza e povertà. Ed. Il Saggiatore 2013; 17-9.

"Ciao Amedeo, hai scritto più di 150 lavori e ne riporto solo uno, perdonami." 